Research Article

\title{
Sample Entropy Analysis of Horses with and without Lameness at Trot before and after Sedation
}

\author{
Jing Zhao $\mathbb{D}^{1},{ }^{1}$ Dan B. Marghitu, ${ }^{1}$ Amey Rane, ${ }^{1}$ and John Schumacher ${ }^{2}$ \\ ${ }^{1}$ Mechanical Engineering Department, Auburn University, Auburn 36849, AL, USA \\ ${ }^{2}$ Department of Clinical Sciences, College of Veterinary Medicine, Auburn University, Auburn 36849, AL, USA \\ Correspondence should be addressed to Jing Zhao; jzz0053@auburn.edu
}

Received 26 May 2020; Revised 20 June 2020; Accepted 25 June 2020; Published 13 July 2020

Guest Editor: Carlos Llopis-Albert

Copyright (c) 2020 Jing Zhao et al. This is an open access article distributed under the Creative Commons Attribution License, which permits unrestricted use, distribution, and reproduction in any medium, provided the original work is properly cited.

The horses were trotted in a straight line, and the acceleration data were recorded from the movement of the horse's head. Inertial sensors were used to collect the information. The sample entropy was used to characterize the dynamics of horse gait. The gait of sound and lame horses was compared, and the sound horses had smaller values of the sample entropy. The effects of sedative detomidine on the gait of lame horses were also examined. The values of sample entropy for sedated horses were smaller than the values of the sample entropy for untreated horses. The sample entropy can provide important information about the dynamics of the equine gait.

\section{Introduction}

The concept of entropy originated from information technology and was introduced to quantify the amount of information obtained in communication signal [1]. The approximate entropy (ApEn) has been developed to classify complex systems that include both deterministic and stochastic processes [2]. The approximate entropy was not suitable for the analysis of the noisy data that are prevalent in biological studies, and inconsistent results were produced. As a modification of the ApEn, the sample entropy (SampEn) was designed to increase the accuracy of ApEn over a broad range of conditions [3]. The sample entropy has been useful in the study of the postural control and the center of pressure displacements $[4,5]$. Stergiou et al. adopted entropy to measure the change in postural control for an infant with cerebral palsy and found that entropy values decreased significantly from prop sitting to beginning independent sitting [6]. Jia et al. reported that sample entropy of the functional connections of brain regions in healthy subjects decreased with advancing age [7]. A smaller value of the SampEn also indicates more self-similarity in dataset or less noise. The sample entropy has higher value for more complicated time series. For cardiovascular time series, one fundamental hypothesis is that healthy subjects have more complicated time series than cardiovascular patients [8].

Lameness in horses causes serious economic losses to the equine industry [9]. Detection of lameness is not difficult when lameness is moderate to severe, but confusion appears when lameness is subtle. There are disagreements between equine clinicians during subjectively evaluating lameness due to diversity of skills developed for lameness recognition. Clinicians are not able to capture small changes in locomotion pattern because of the relatively low image capturing frequency of human eye [10]. Detomidine, a commonly used sedative in horses, is often administered to unruly horses to facilitate lameness examination $[11,12]$. However, there is a concern among veterinarians about the effect of sedation on movement patterns of horses $[13,14]$. Many clinicians are reluctant to use sedative during lameness examination as they believe it might affect the identification of subtle lameness because of the inherent analgesic property of this drug $[15,16]$. The effect of sedation on the gait of lame horses has been studied using vector sums of maximum and minimum head height and the sum of hip drop and hip hike [17].

Multiple methods have been initiated for studying the nonlinear dynamics of gait and human motion which may include model development, dynamic formulation, and 
simulation solver [18]. The nonlinear dynamics equations are directly integrated or differentiated. The simulation solver demands experimental motion data and optimization-based methods. For the repeated motion of the hip during walking, an analytical approach has been proposed to obtain solution for nonlinear oscillators [19]. Optimal auxiliary functions are employed to approximate the analytical solution of the system. The deep learning technique has been used for the gait patterns [20]. With the help of deterministic learning algorithms, detailed information about gait dynamics can be produced. The authors applied the knowledge fusion strategy for gait aspects.

Equine locomotion involves rhythmic and coordinated patterns of body and limb segments [21]. An equine body system can be considered as a dynamic system, and the changes occurring in the body due to lameness can be analyzed according to dynamic laws. For the last two decades, using inertial sensors and high-speed camera capturing devices, researchers have studied the dynamic locomotion of the horse.

The concept of entropy was initiated from the energy loss during combustion and not converted into functional work. In thermodynamics, any event in an isolated system will make the entropy larger. The topological entropy estimates, in time, the progression of detectable orbits for an iterated function describing the dynamical system. For a stochastic system, the entropy is the average rate at which the information is produced. The proposed definition of entropy (SampEn) is a chosen option in our study.

The objective of this paper was to implement the SampEn for the equine gait. First, we studied the trot of sound and lame horses. Next, we compared the gait of unsedated lame horses with their gait when sedated with detomidine. The vertical movement data of the horse's head were used for this study. To our best knowledge, the use of SampEn on the analysis of horse gait was not studied. The SampEn can be utilized for the dynamic analysis of the horse gait.

\section{Materials and Methods}

Ten horses that were determined by subjective evaluation from the investigator to be lame and eight to be nonlame were enrolled in this study. The mean weight of the horses was $460 \pm 50 \mathrm{~kg}$. In the first study, the gait from sound horses and lame horses was compared by calculating SampEn. Horses were trotted for at least 25 consecutive strides during each of 10 random trials while wearing sensors.

In the second study, the effect of sedative detomidine on gait of ten lame horses was studied using two groups: Group 1 (no treatment, control group) and Group 2 (horses administered $10 \mathrm{mg}$ of detomidine HCL). Horses were randomly assigned to two groups [17]. Lame horses were trotted about 25 consecutive strides every 5 minutes for 45 minutes. This study was approved by Auburn University's Animal Care and Use Committee.

Two inertial sensors were attached noninvasively to the horse as shown in Figure 1. A single-axis accelerometer was

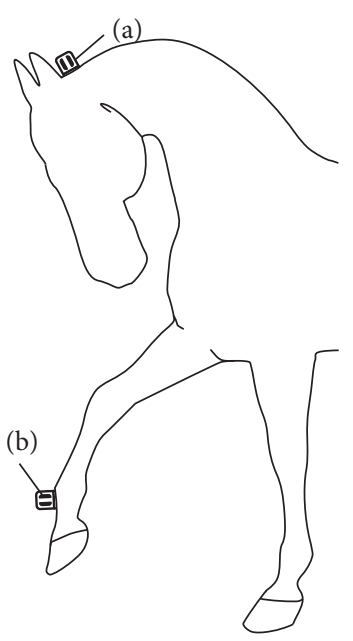

FIGURE 1: Inertial sensors attached noninvasively to the horse: (a) uniaxis vertical accelerometer head sensor and (b) uniaxial gyroscope right forelimb sensor.

mounted on the head halter to measure the vertical acceleration of the head. A single-axis gyroscope was attached to dorsal surface of the region between the metacarpophalangeal joint and coronary band of the right forelimb to measure the rotation of the foot. The signal from sensors was transmitted to a tablet $\mathrm{PC}$ via Bluetooth. The raw data collected from the sensors were processed in MATLAB.

The data were in tsv format with a unique 32-digit hex address. The raw output value in this file was digitized (8-bit) from the voltage value ( $+/-5$ volts). A conversion factor was used to get the actual acceleration data. Figure 2 shows a simultaneous plot of vertical head acceleration and right forefoot angular velocity. Stride split was achieved by using the information from the gyroscope located at the right forelimb. Figure 3 shows the head acceleration data gathered from ten strides.

2.1. Sample Entropy. Acceleration data for each stride were time normalized to 50 data points. The whole motion cycle with 20 strides was time normalized with 1000 data points. This data length is considered sufficient for the entropy analysis [22]. After signal is segmented into strides and normalized, the entropy calculation was carried out. The SampEn gives us the probability of two data that are close (predetermined tolerance level) in $m$ dimensional space and remain close in $m+1$ dimensional space. Larger is the probability less is the SampEn value and vice versa.

The sample entropy of a time series $x_{1}, x_{2}, \ldots x_{N}$ of length $N$ with zero mean and unit standard deviation is defined by

$$
\operatorname{SampEn}(N, m, r)=-\log \left(\frac{A(r)}{B(r)}\right)
$$

where $m$ is the length of the subsequences of the time series; $r$ is the maximum distance (or tolerance) between two subsequences of the same length $m ; A(r)$ is the total number 


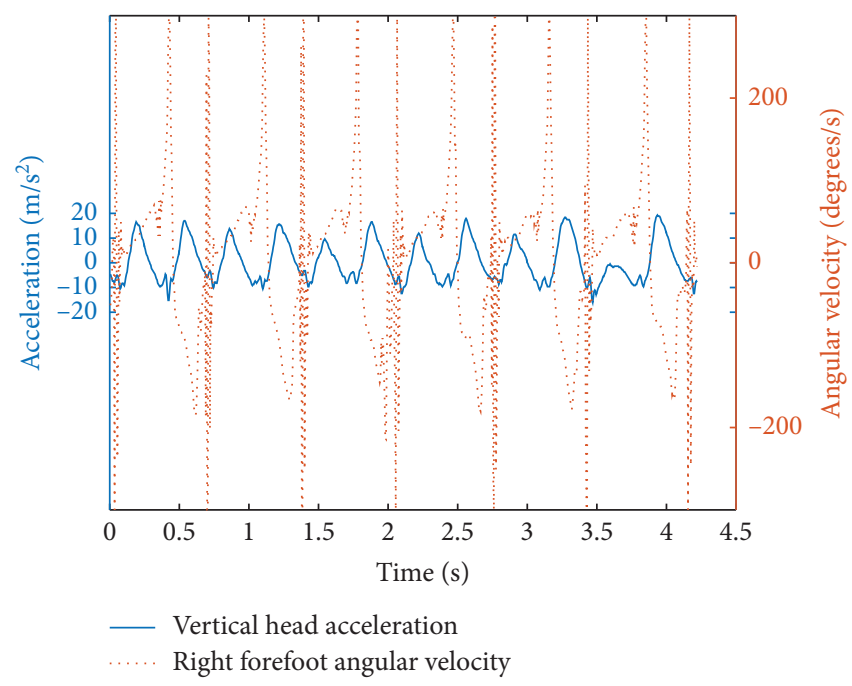

FIGURE 2: Vertical head acceleration and right forefoot angular velocity.

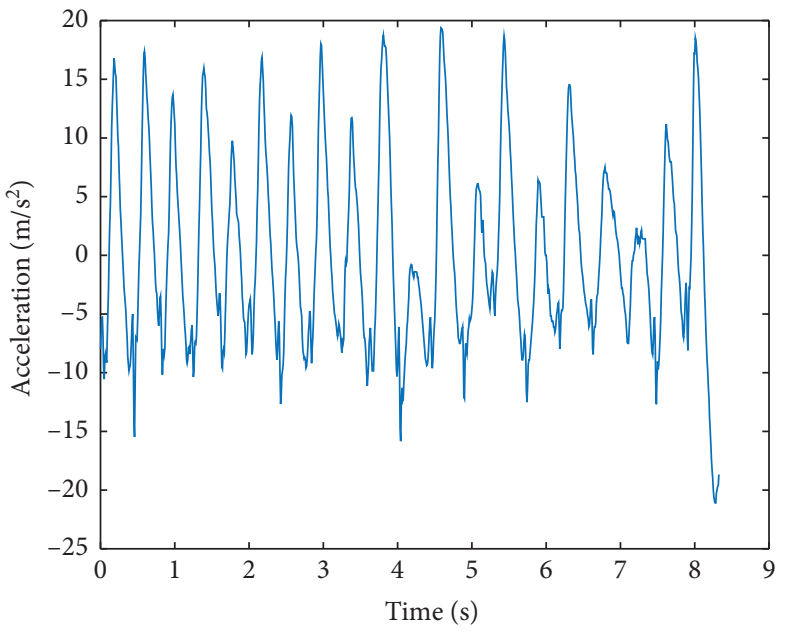

FIgURE 3: Head acceleration for ten strides.

of subsequences in a $m+1$ dimensional phase space that are within the distance $r$; and $B(r)$ is the total number of subsequences in a $m$ dimensional phase space that are within the tolerance $r$. The complexity level of two signals could be determined from this method. For the computation of the SampEn, the guideline detailed in Ramdani et al. was followed [23].

Sample entropy is found to be closely related to selection of $m$ and $r$ for short datasets. The embedding dimension $m$ and the tolerance $r$ are selected according to Lake et al. [24]. The procedure is based on the convergence criterion which estimates $m$ leading to the selection of $r$. The median SampEn is calculated for all datasets as a function of $r$ for $m=1,2,3,4,5$.

Figure 4 shows that the entropy values start converging when $m$ is equal to 3 which lead us to select $m=3$. Next step is to compute the median of maximum relative error as a function of $r$ for $m=3$. The value of $r$ is then estimated to give maximum relative error no higher than 0.05 .

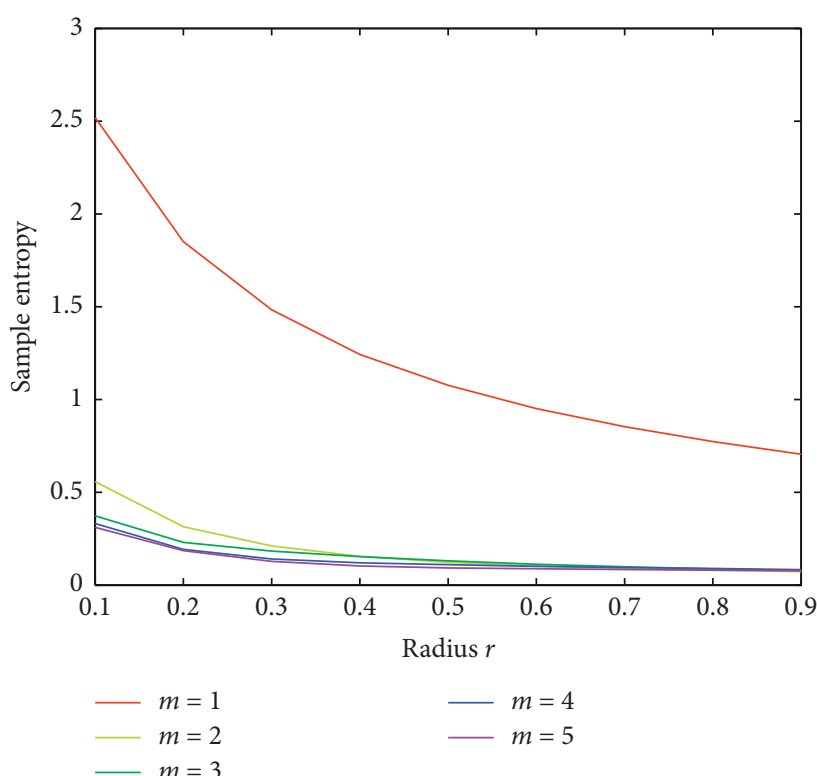

Figure 4: Median SampEn as a function of $m$ and $r$.

2.2. Statistical Analysis. The difference of SampEn between sound and lame horses was compared with $t$ test. The SampEn values were subjected to a log transformation to ensure the homogeneity of variance. To test the effect of detomidine on SampEn, linear mixed effects model $Y=X \beta+Z \mu+e$ was performed, where $Y$ was the SampEn values, $X$ was the treatment design matrix (treatment with detomidine or no treatment; data collection at 5, 10, 15, 20, $25,30,35,40$, and 45 minutes), $\beta$ was the vector of fixed treatment effects, $Z$ was the subject design matrix (horse), $\mu$ was a vector of random subject-specific effects, and $e$ was a vector of random experimental error. The normality of the data was tested using the Shapiro-Wilk test. First-order autoregression has been used as the correlation structure. If a significant influence was found, paired $t$ tests were performed to determine where significant differences were present. The significance level was set at $p<0.05$. Statistical analyses were performed using SAS (SAS Studio 3.8, SAS Institute Inc., Cary, NC, USA.).

\section{Results}

When applied fast Fourier transform, no major differences were found between lame and sound horses (Figure 5). The phase planes are affected by the duration of the detomidine treatment but there are no limit cycles or clear attractors (Figure 6). For a periodic solution, the phase plane is a closed curve. For the return maps, there are differences between $5 \mathrm{~min}$ and $45 \mathrm{~min}$ following detomidine treatment but the systems are not periodic or quasiperiodic (Figure 7). The return map of a periodic system consists of a finite set of points. For a quasiperiodic system, a closed orbit tends to display on the return map. If a system has chaotic motion, an infinite number of points with a well-defined structure usually make up the return map. We cannot find distinct points, segments, or curves on the return maps. 


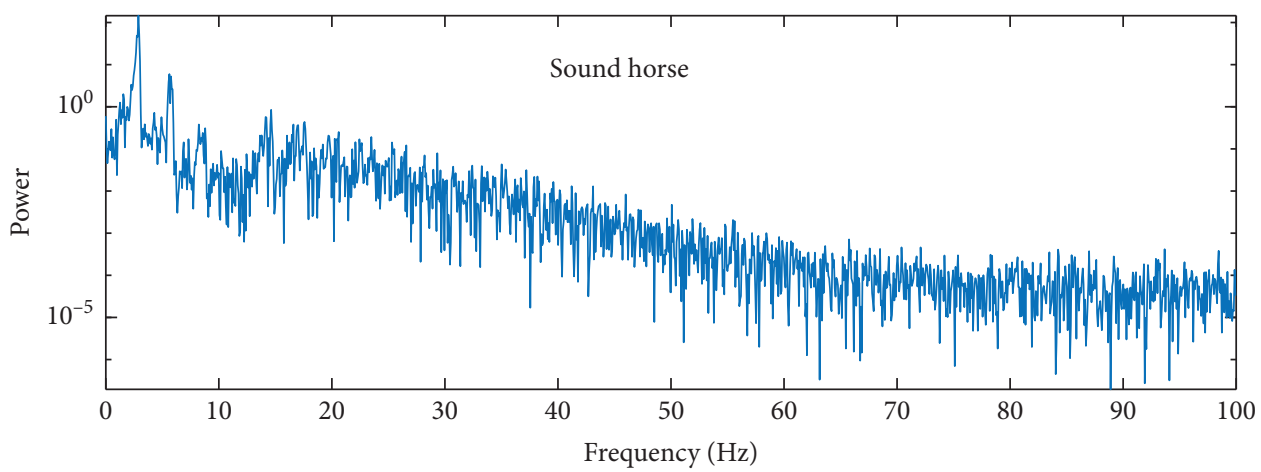

(a)

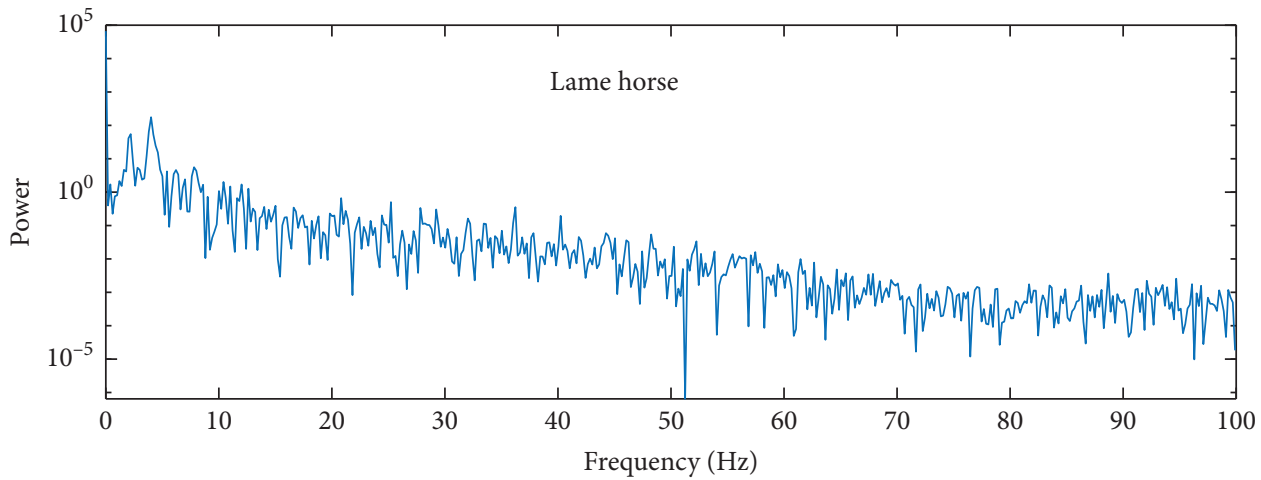

(b)

FiguRE 5: Fast Fourier transform for (a) a sound horse and (b) a lame horse.

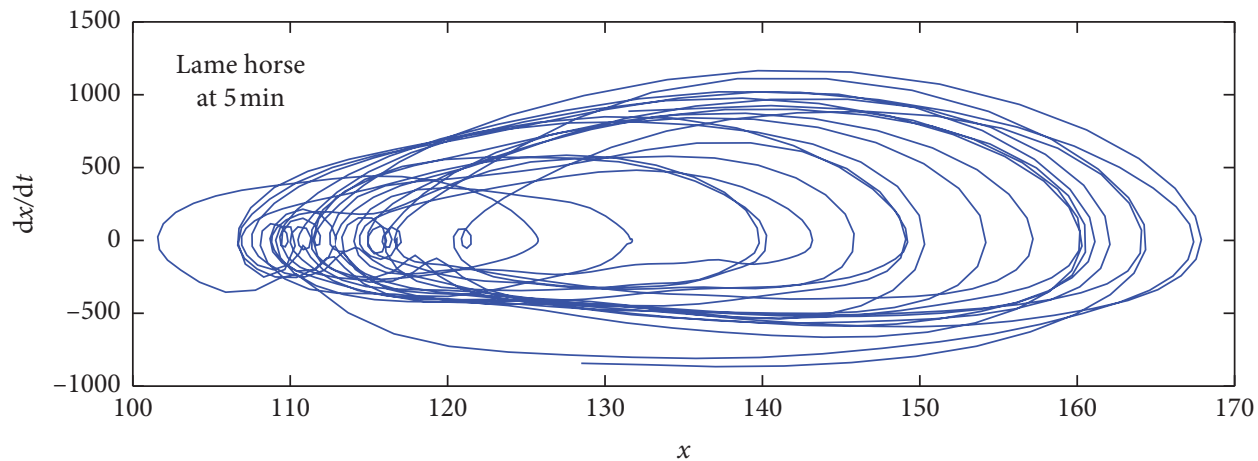

(a)

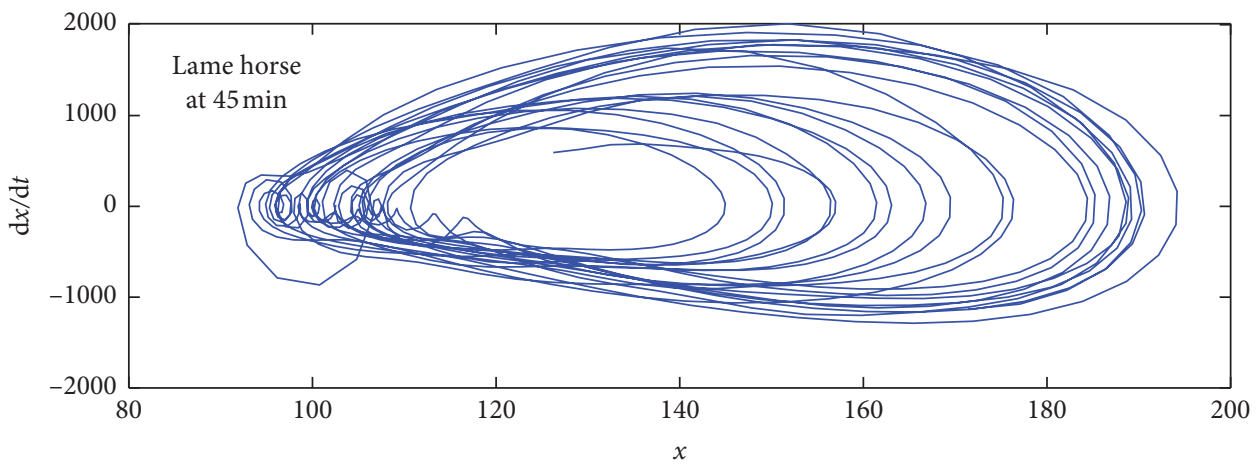

(b)

FIgURE 6: Phase plane for a lame horse at (a) $5 \mathrm{~min}$ and (b) $45 \mathrm{~min}$ following treatment with detomidine. 


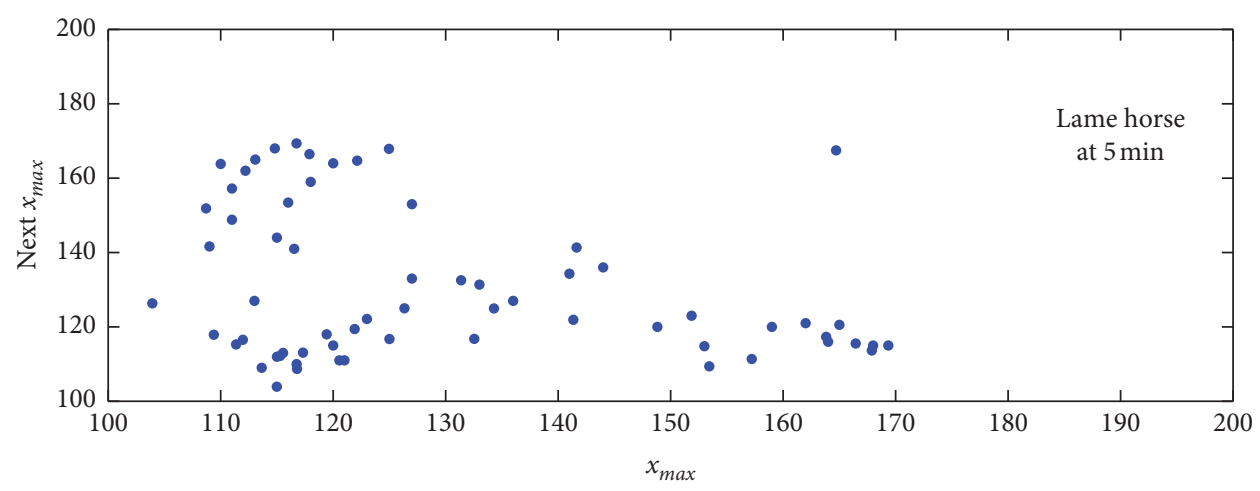

(a)

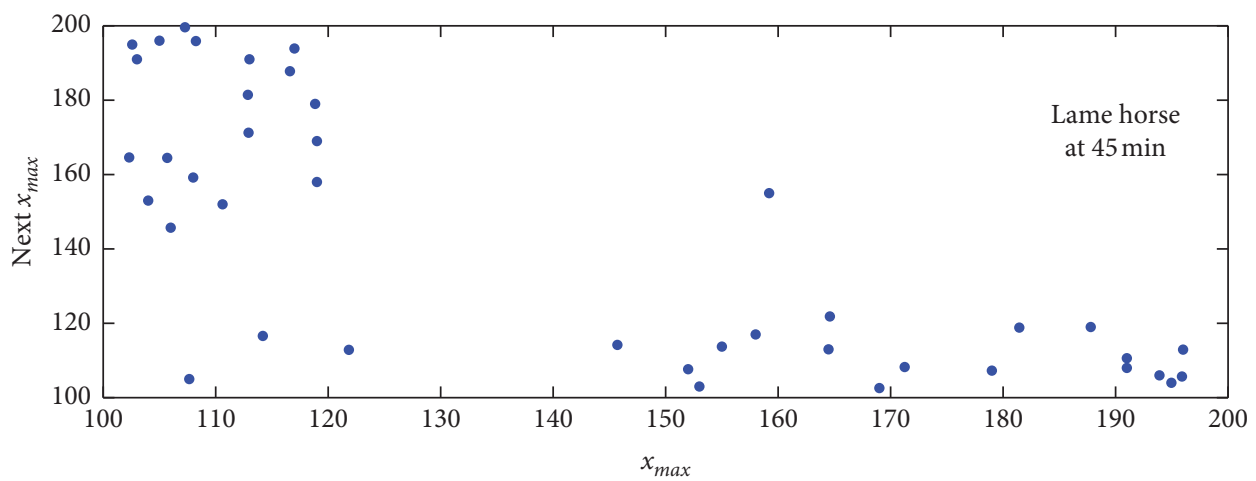

(b)

FIgURE 7: The return map for a lame horse at (a) $5 \mathrm{~min}$ and (b) $45 \mathrm{~min}$ following treatment with detomidine.

Gait from sound horses was associated with significantly smaller SampEn than lame horses $(p<0.0001)$ (Figure 8 ).

Treatment with detomidine had a significant effect on the SampEn of lame horses compared with no treatment $(p=0.0225)$ (Figure 9). At the $15 \mathrm{~min}$ data collection, the SampEn was significantly lower after treatment with detomidine compared with no treatment $(p=0.0438)$. No significant differences were found at other times of data collection.

\section{Discussion}

Based on the results of this study, the sample entropies of lame horses are larger than that of sound horses. The sample entropies were significantly smaller at $15 \mathrm{~min}$ after the intravenous administration of $10 \mathrm{mg}$ detomidine compared with no treatment. Our results are consistent with the results of Buchner et al. which observed a decrease of head height and increase of stride duration and stride length after detomidine sedation, with the maximum effect of detomidine at $15 \mathrm{~min}$ recording [11].

The investigation of sample entropy may reveal important information about the characteristics of locomotion pattern. Higher entropy values are associated with a functional decline of the locomotion control system, indicating a greater need for adaptability to perturbations in the system [5]. In contrast, the signal emanated from a system with low entropy values generates less additional information over

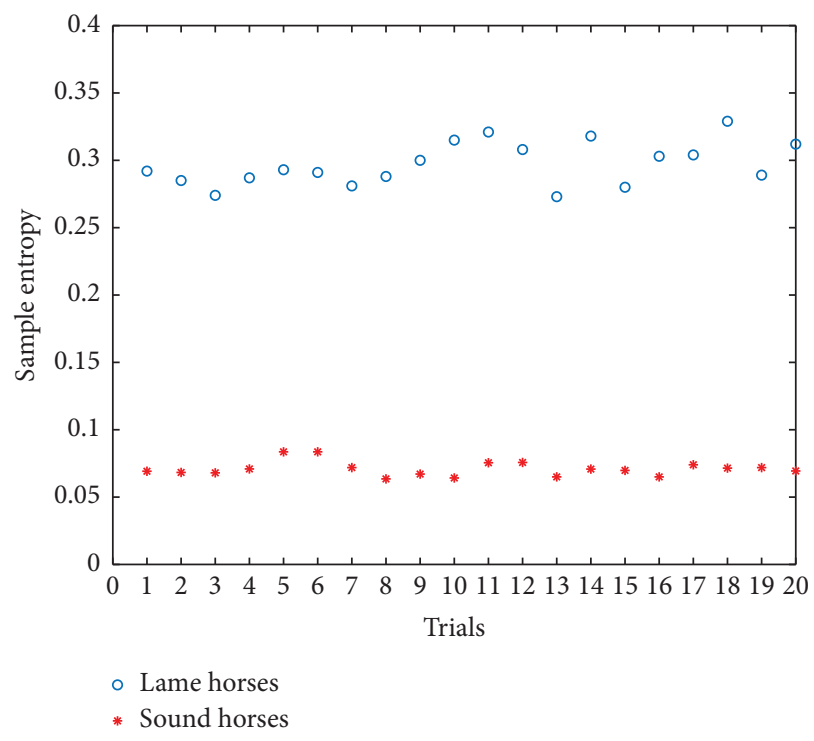

Figure 8: Comparison of SampEn between sound and lame horses.

time and is more predictable [25]. The less complex gait was observed in the sound horses in this study.

It has been reported that gait complexity of human adults walking on a treadmill increased with increasing walking speed, possibly related to the mechanism of 


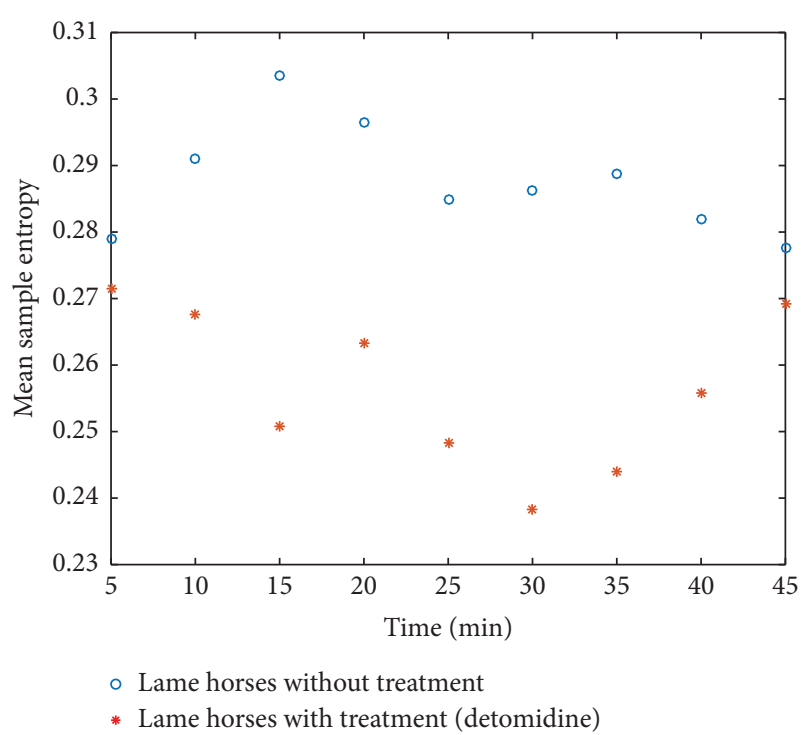

Figure 9: Mean SampEn of ten horses at 5, 10, and every 5 min over a total period of $45 \mathrm{~min}$ following no treatment and treatment with detomidine.

movement control [26]. In this study, however, horses were trotted by a single handler for consistency of speed. Evaluation of the effect of speed on sample entropy of horses should be considered for future study to help understanding the control system in horses. Furthermore, future studies of different gait of sound horses would benefit from using sample entropy as a new measurement variable.

\section{Conclusion}

In spite of achievements in the area, there is no common measure to evaluate the dynamics of animal and human locomotion. In the present study, we investigated the equine gait of sound and lame horses. By comparison, smaller SampEn was identified on sound horses. Moreover, we observed the effect of detomidine on gait of lame horses. The sample entropy values for horses with detomidine were smaller than the SampEn of horses that did not receive a treatment. In the present work, we have shown that, using an appropriate methodology, the SampEn algorithm was able to characterize the dynamic features of equine gait. The technique in this study gives the clinical team a useful measure of the gait. It is expected that this research will have straight implementation to the analysis of gait in humans and animals.

\section{Data Availability}

The data used to support the findings of this study are included within the article.

\section{Conflicts of Interest}

The authors declare that there are no conflicts of interest regarding the publication of this paper.

\section{References}

[1] C. E. Shannon, "A mathematical theory of communication," Bell System Technical Journal, vol. 27, no. 3, pp. 379-423, 1948.

[2] S. M. Pincus, "Approximate entropy as a measure of system complexity," Proceedings of the National Academy of Sciences, vol. 88, no. 6, pp. 2297-2301, 1991.

[3] J. S. Richman and J. R. Moorman, "Physiological time-series analysis using approximate entropy and sample entropy," American Journal of Physiology-Heart and Circulatory Physiology, vol. 278, no. 6, pp. H2039-H2049, 2000.

[4] A. V. Lubetzky, D. Harel, and E. Lubetzky, "On the effects of signal processing on sample entropy for postural control," PLoS One, vol. 13, no. 3, pp. 1-15, 2018.

[5] C. Hansen, Q. Wei, J. S. Shieh, P. Fourcade, B. Isableu, and L. Majed, "Sample entropy, univariate, and multivariate multi-scale entropy in comparison with classical postural sway parameters in young healthy adults," Frontiers in $\mathrm{Hu}$ man Neuroscience, vol. 11, p. 206, 2017.

[6] N. Stergiou, R. T. Harbourne, and J. T. Cavanaugh, "Optimal movement variability," Journal of Neurologic Physical Therapy, vol. 30, no. 3, pp. 120-129, 2006.

[7] Y. Jia, H. Gu, and Q. Luo, "Sample entropy reveals an agerelated reduction in the complexity of dynamic brain," Scientific Reports, vol. 7, p. 7990, 2017.

[8] C. Chen, Y. Jin, I. L. Lo et al., "Complexity change in cardiovascular disease," International Journal of Biological Sciences, vol. 13, no. 10, pp. 1320-1328, 2017.

[9] L. Bourebaba, M. Röcken, and K. Marycz, "Osteochondritis dissecans (OCD) in horses-molecular background of its pathogenesis and perspectives for progenitor stem cell therapy," Stem Cell Reviews and Reports, vol. 15, no. 3, pp. 374390, 2019.

[10] K. W. Hinchcliff, A. J. Kaneps, and R. J. Geor, Equine Sports Medicine and Surgery E-Book, Elsevier Health Sciences, Amsterdam, Netherlands, 2013.

[11] H. H. F. Buchner, P. Kübber, E. Zohmann, and C. Peham, "Sedation and antisedation as tools in equine lameness examination," Equine Veterinary Journal, vol. 31, no. S30, pp. 227-230, 1999.

[12] H. K. DiMaio Knych and S. D. Stanley, "Pharmacokinetics and pharmacodynamics of detomidine following sublingual administration to horses," American Journal of Veterinary Research, vol. 72, no. 10, pp. 1378-1385, 2011.

[13] F. J. López-Sanromán, R. Holmbak-Petersen, M. Varela, A. M. Del Alamo, and I. Santiago, "Accelerometric comparison of the locomotor pattern of horses sedated with xylazine hydrochloride, detomidine hydrochloride, or romifidine hydrochloridefidine hydrochloride," American Journal of Veterinary Research, vol. 74, no. 6, pp. 828-834, 2013.

[14] F. J. López-Sanromán, S. De La Riva Andrés, R. HolmbakPetersen, M. Pérez-Nogués, P. Forés Jackson, and M. Santos González, "An accelerometric measure of the gait pattern in horses after the administration of sublingual detomidine," Research in Veterinary Science, vol. 97, no. 2, pp. 391-396, 2014.

[15] R. Pilsworth and S. Dyson, "Where does it hurt? Problems with interpretation of regional and intra-synovial diagnostic analgesia," Equine Veterinary Education, vol. 27, no. 11, pp. 595-603, 2015.

[16] A. E. Fürst, "Diagnostic anesthesia," in Equine Surgery, J. A. Auer and J. A. Stick, Eds., pp. 998-1015, W.B. Saunders, Saint Louis, 2012. 
[17] J. Taintor, F. DeGraves, and J. Schumacher, "Effect of tranquilization or sedation on the gait of lame horses," Journal of Equine Veterinary Science, vol. 43, pp. 97-100, 2016.

[18] M. Ezati, B. Ghannadi, and J. McPhee, "A review of simulation methods for human movement dynamics with emphasis on gait," Multibody System Dynamics, vol. 47, no. 3, pp. 265-292, 2019.

[19] D. Mihut and N. Herisanu, "A new analytical approach to investigate human gait dynamics," ITM Web of Conferences. EDP Sciences, vol. 29, Article ID 02004, 2019.

[20] M. Deng, T. Fan, J. Cao, S. Y. Fung, and J. Zhang, "Human gait recognition based on deterministic learning and knowledge fusion through multiple walking views," Journal of the Franklin Institute, vol. 357, no. 4, pp. 2471-2491, 2019.

[21] E. Barrey, "Methods, applications and limitations of gait analysis in horses," The Veterinary Journal, vol. 157, no. 1, pp. 7-22, 1999.

[22] J. M. Yentes, N. Hunt, K. K. Schmid, J. P. Kaipust, D. McGrath, and N. Stergiou, "The appropriate use of approximate entropy and sample entropy with short data sets," Annals of Biomedical Engineering, vol. 41, no. 2, pp. 349-365, 2013.

[23] S. Ramdani, B. Seigle, J. Lagarde, F. Bouchara, and P. L. Bernard, "On the use of sample entropy to analyze human postural sway data," Medical Engineering \& Physics, vol. 31, no. 8, pp. 1023-1031, 2009.

[24] D. E. Lake, J. S. Richman, M. P. Moorman, J. R. Fin, and J. R. Moorman, "Sample entropy analysis of neonatal heart rate variability," American Journal of Physiology-Regulatory, Integrative and Comparative Physiology, vol. 283, no. 3, pp. R789-R797, 2002.

[25] J. H. Hollman, M. K. Watkins, A. C. Imhoff, C. E. Braun, K. A. Akervik, and D. K. Ness, "Complexity, fractal dynamics and determinism in treadmill ambulation: implications for clinical biomechanists," Clinical Biomechanics, vol. 37, pp. 91-97, 2016.

[26] G. S. Walsh and Z. Taylor, "Complexity, symmetry and variability of forward and backward walking at different speeds and transfer effects on forward walking: implications for neural control," Journal of Biomechanics, vol. 97, p. 109377, 2019. 